\title{
Theoretical aspects and practical implications of the heuristic drift SOL model
}

\author{
R.J. Goldston ${ }^{\mathrm{a} *}$ \\ ${ }^{a}$ Princeton Plasma Physics Laboratory, Princeton NJ 08543 USA
}

The heuristic drift (HD) model for the tokamak power scrape-off layer width provides remarkable agreement in both absolute magnitude and scalings with the measured width of the exponential component of the heat flux at divertors targets, in low gas-puff H-Mode tokamaks. This motivates further exploration of its theoretical aspects and practical implications. The HD model requires a small non-ambipolar electron particle diffusivity $\sim 10^{-2} \mathrm{~m}^{2} / \mathrm{sec}$. It also implies large parallel heat flux in ITER and suggests that more radical approaches will be needed to handle the $\sim 20 \mathrm{GW} / \mathrm{m}^{2}$ parallel heat flux expected in Demo. Remarkably, the HD model is also in good agreement with recent near-SOL heat flux profiles measured in a number of limiter L-Mode experiments, implying ubiquity of the underlying mechanism. Finally, the HD model suggests that the H-mode and more generally Greenwald density limit may be caused by MHD instability in the SOL, rather than originating in the core plasma or pedestal. If the SOL width in stellarators is set by magnetic topology rather than by drifts, this would be consistent with the absence of the Greenwald density limit in stellarators.

PACS: 52.40.Hf, 52.55.Fa, 52.55.Rk

PSI-21 Keywords: Density limit, Divertor, Divertor plasma, Edge plasma

* Corresponding author address: Princeton Plasma Physics Laboratory, Princeton

University, P.O. Box 452 - MS41, Princeton, NJ 08543 USA

* Corresponding author E-mail: goldston@pppl.gov 
Presenting author: Robert J. Goldston

Presenting author e-mail: goldston@pppl.gov

Theoretical aspects and experimental implications of the heuristic drift SOL model

\section{Introduction}

The Heuristic Drift (HD) model [1] for the scrape-off-layer in low-gas-puff H-Mode tokamaks exhibits remarkable agreement with the exponential power width, $\lambda_{q}$, measured in a wide range of tokamak experiments [2, 3, 4]. This model balances $c_{s} / 2$ parallel flows against $\operatorname{grad} B$ and curv $B$ drifts to find $\lambda_{q} \sim 2(a / R) \rho_{p}$, similar to the orbit shift of collisionless passing ions. The edge temperature needed to evaluate $\rho_{p}$ is derived from Spitzer parallel electron thermal conduction, on the assumption that while the density channel in the SOL is established by ion dynamics, the parallel heat flux is confined to this channel and carried by electrons [1]. The success of the HD model both with respect to the absolute value of the power scrape off width (Figure 1) and its scalings with $B_{T}, q_{c y l}, P, R$ and $a / R$ (Figure 2), suggests that its theoretical aspects should be further pursued (Section 2), as well as its practical implications for ITER and Demo (Section 3), its implications for limiter tokamaks (Section 4) and its implications for the tokamak density limit (Section 5).

\section{Theoretical Aspects}

It has already been noted [1] that order-unity poloidal pressure variation is required to obtain net ion drift flow across the separatrix of order the ion drift flux. Chankin and Coster [5] emphasize that on closed field lines an up-down ion pressure asymmetry is required to obtain this flow. They find that for poloidally constant temperature, a Boltzmann electron distribution results in a poloidal electric field and so radial $\boldsymbol{E} x \boldsymbol{B}$ drift that eliminates any net 
electron transport. For $T_{e}=T_{i}$, this drift halves the top-bottom ion pressure asymmetry required for a given net ion flow. This result was also shown in [6].

As noted in [5], and as follows from the fact that the full poloidal integrals in the plasma that are required for the proof of net zero electron drift transport are interrupted by material surfaces, lack of ambipolar transport does not present a problem for the HD model on open field lines, but does raise the question as to how quasi-neutrality is preserved within closed flux surfaces in this model. Anomalous electron thermal diffusivity of order $1 \mathrm{~m}^{2} / \mathrm{sec}$ has been deduced from power balance at the separatrix in both ASDEX-U [7] and DIII-D [8]. Combining the net ion loss current calculated in the HD model, equation (2) of [1], with a density gradient scale length of $\lambda_{q}$, one can deduce the requirement for a non-ambipolar electron particle diffusivity $D_{e, N A}=2^{1 / 2} T_{s e p} \lambda_{q} /\left[e B \pi R\left(1+\kappa^{2}\right)^{1 / 2}\right]$ to balance the outgoing ion current. For $T_{\text {sep }}=80 \mathrm{eV}, \lambda_{q}=3 \mathrm{~mm}, B=2 \mathrm{~T}, R=1.6 \mathrm{~m}, \kappa=1.7, D_{e, N A}=0.016$

$\mathrm{m}^{2} / \mathrm{sec}<<\chi_{e}$. Such a small amount of non-ambipolar electron diffusive loss (or transport due to radial electric fields of order $T_{e} / e \lambda_{q}$ ) could be due to externally induced magnetic ergodicity near the separatrix or to turbulence that couples momentum across the separatrix. Thus quasi-neutrality does not present a conceptual issue for the HD model even on closed field lines close to the separatrix, since non-ambipolar anomalous electron radial current could balance the radial ion drift loss, resulting in overall ambipolarity.

\section{Implications for ITER and Demo Heat-Flux Dissipation}

The agreement of experimental results with the HD model is encouraging for using it to extrapolate to devices well beyond the parameter range of current experiments, because the HD model is consistent with the constraints of plasma physics, while a regression fit to the data need not be. Furthermore, if both model and experiment give closely similar extrapolations this increases confidence in the projection. It is also encouraging for this use 
that of the 23 combinations of parallel and perpendicular transport mechanisms and three marginal stability criteria from which Fundamenski et al. [9] derived scalings for the SOL width, only the examples corresponding to the drift and flow physics of the HD model give the very weak $R$ scaling observed in experiment. These are 1D+ and 1D, the latter of which corresponds to sheath-limited electron heat loss. Two others (1C and $\left.1 \mathrm{C}^{*}\right)$ may be barely within the experimental error on $R$ scaling, but are far too weak in their scalings with $B_{p}$ [3].

When we extrapolate to ITER, we project a very narrow SOL layer, which itself has also elicited a challenge based on what was perceived to be the necessary upstream pressure [10]. Consistent with [10] we assume that ITER will release $100 \mathrm{MW}$ of transport power across the separatrix, at a line average density of $110^{20} / \mathrm{m}^{3}$. Using $B / B_{p}=3.4$ at the outer midplane (OMP), $2 / 3$ of the power going to the outer divertor, and $I_{q, \text { oMP }}=\square 1 \mathrm{~mm}$ (from either HD-based or empirical extrapolation to ITER) the average parallel power density along a field line connecting the OMP with the outer divertor target is an impressive $5.5 \mathrm{GW} / \mathrm{m}^{2}$. This is problematic both due to the difficulty of dissipating so much heat flux, and due to the fact that the residual heat flux to the divertor surface is given by the residual $q_{/ /}$multiplied by $\sin \alpha$, with the value of $\alpha$ limited from below due to mechanical asymmetries in the divertor.

Taking a separatrix density of $3.310^{19} / \mathrm{m}^{2}, 1 / 3$ of the line average, and including a factor $f_{\text {power }}$ to represent the fraction of $q_{/ /}$dissipated near the divertor plate through radiation, charge-exchange and radial transport, one can solve the generalized 2-point model [11] as a function of $f_{\text {power }}$ (Figure 3). As can be seen, dissipation in excess of $95 \%$ will be required to access acceptable target temperatures in ITER. For a $2^{\mathrm{O}}$ strike angle this value of $f_{\text {power }}$ would also reduce the perpendicular heat flux to $\sim 10 \mathrm{MW} / \mathrm{m}^{2}$. Lower $f_{\text {power }}$ would lead to higher temperatures ( $600 \mathrm{eV}$ using $f_{\text {power }}=0.5$ as assumed by ITER) and higher target heat fluxes, and so would result in the build-up of unacceptable plasma contamination and/or damage to plasma-facing components. However these conditions are not impossible from the point of 
view of the physics of the main plasma and SOL as is argued in [10]. Note in particular that the upstream plasma pressure associated with $T=600 \mathrm{eV}$ and $n=3.310^{19} / \mathrm{m}^{3}$ is small compared with the expected pedestal pressure, which is projected to be about 10x this value.

Extrapolating to a Demo with the size of ITER but with $5 \mathrm{x}$ higher fusion power and $\mathrm{Q}$ $=25$ rather than 10 , the parallel heat flux is projected to be a daunting $18.5 \mathrm{GW} / \mathrm{m}^{2}$. With a highly dissipative divertor this would require an upstream temperature of about $400 \mathrm{eV}$, which for $1.5 \mathrm{x}$ the separatrix density of ITER corresponds to a pressure of $6300 \mathrm{~Pa}$. For a divertor strike angle of $2^{\mathrm{O}}$, and a maximum perpendicular heat flux of $10 \mathrm{MW} / \mathrm{m}^{2} f_{\text {power }}$ would need to be in excess of $98 \%$. This scenario essentially requires fully detached divertor operation, presumably without deleterious effects on confinement. One could ask if it is appropriate to use the HD model for the upstream parallel heat flux in such detached conditions. If flow remains $\sim c_{s} / 2$ then the model is appropriate, but it does provide some encouragement that if flow along the SOL can be choked, such that the hot ions from the bulk plasma have an increased residence time in the SOL, even the upstream SOL width will grow. This physics requires modeling with 2D divertor codes.

To achieve full detachment necessitates both power dissipation and momentum balance against a buffer gas at a pressure approaching $6300 \mathrm{~Pa}$. Based on current experimental experience with full detachment, it is clear that the buffer gas needs to be very well confined in the divertor chamber, both geometrically and through incoming plasma flow, something easier to achieve with a condensing vapor than with deuterium and tritium. Atomic lithium vapor in evaporation / redeposition equilibrium with a divertor surface at $~ 950 \mathrm{C}$ could supply both the necessary pressure [12] and adequate stopping power for ions and electrons to establish momentum balance over an acceptable distance [13]. 


\section{Implications for limiter tokamaks (and comparison with experiment)}

On T-10 [14] and TEXTOR [15] it was observed that the apparent parallel heat flux contained both a near and a far scrape-off zone, which could be characterized by a "summed exponential" form: $q_{\|}(r)=q_{\|, 0, \text { near }}(r) \exp \left(-r / \lambda_{\text {near }}\right)+q_{\|, 0, f a r}(r) \exp \left(-r / \lambda_{\text {far }}\right)$. In both cases this was made evident by the heat flux pattern observed on a limiter optimized for a single exponential decay. Similar results on JET [16] raised concerns that this effect should be taken into account in the design of the ITER inner-wall limiter, which is to be used for plasma start-up.

Experiments on COMPASS using a tiltable limiter [17] have confirmed that the surface heat flux pattern is consistent with $q_{\perp}=q_{||} \sin (\alpha)$ for angles down to $\sim 3-5^{\circ}$, and that nonambipolar currents [18], while present, play a sub-dominant role. Subsequent measurements on TCV [19], DIII-D [20] and C-MOD [21] have confirmed the presence of this feature, in some cases via IR limiter measurements and in others on Langmuir probes at the limiter or in the plasma.

The HD model was not developed for L-Mode, limiter plasmas. As discussed in [1] the model clearly does not give the correct global particle confinement time for L-Mode plasmas, because there is more flux across the separatrix than predicted. However it is reasonable that the same drifts and parallel flows would take place within the $S O L$, albeit in a noisier environment and fueled more strongly from the main plasma. In this picture $\lambda_{\text {near }}$ would be determined by HD physics, while $\lambda_{f a r}$ would arise from turbulence. In effect $\lambda_{\text {far }}$ would play a similar role to Eich's $S$ parameter [2] for divertor plasmas.

Since limiter plasmas are generally in the sheath-limited regime, the Spitzer conductivity estimate for $T_{e}$ used in the HD model is not strictly justifiable. However $T_{e}$ comes in only to the $1 / 2$ power, and the edge temperatures measured with probes in COMPASS are close to the calculated Spitzer values using the measured $q_{\|}$. Furthermore, as shown in [9] the overall scaling for the SOL width with HD physics and sheath-limited $T_{e}$ is 
similar. Changes in the heat-transmission factor, $\gamma$, due to non-ambipolar currents [18] play no role in the standard HD calculation, since $T$, which determines the SOL width, is set by Spitzer conductivity. Even in a deeply sheath-limited regime with $T>>T_{\text {Spitzer }}$ (not seen in these experiments) $\lambda_{q}$ would scale only as $\left(n_{\text {sep }} \gamma\right)^{-1 / 4}$.

Another difference compared with divertor plasmas is that one does not expect high recycling at a limiter, and so parallel flows could be expected to be higher than in a divertor case, but certainly no greater than $c_{s}$. This effect goes in the opposite direction. None of these effects should be as large as a factor of 1.5, so one should expect the HD model to have somewhat poorer agreement for these conditions than for H-Mode divertor plasmas, but be representative of the trends. In ASDEX-U and JET divertor L-Mode plasmas [19] the regression fits give scalings that are similar to the HD model, but significant differences are seen in the variation with power and size (figures 3 and 6 ). The observed overall $\sim 2$ times greater width could be due to greater choking of flow in the divertor under ASDEX-U LMode conditions and so a longer ion residence time in the SOL.

Figure 4 shows the result of plotting data for $\lambda_{\text {near }}$ from "summed exponential" fits, kindly provided by the C-MOD [22], COMPASS [17, 18], DIII-D [21], JET [16] and TCV [20] teams, compared against the HD result. Also included are single points for T-10 [14] and TEXTOR [15]. The similarity in trend and in absolute magnitude to the divertor data in Figure 1 is striking, supporting the hypothesis that the same mechanisms are at play. Of practical importance, the overlap of measured data from large machines such as JET with small ones such as COMPASS and TCV indicates that the width of the narrow feature in limiter plasmas scales weakly with system size, as was found for $\lambda_{q}$ in divertor plasmas. This is consistent with the fundamental result of the HD model, which is that the basic scaling of the near SOL width is $(a / R) \rho_{p}$, an intensive scaling, as opposed to the scaling with extensive 
variables expected in other SOL models. These results have led to design changes for the ITER inner-wall limiter.

\section{Implications for the tokamak density limit}

It is interesting to derive the MHD $\alpha$ parameter in the SOL based on the assumption that the pressure gradient scale length at the separatrix is approximately equal to the experimentally measured $\lambda_{q}$. If we use Spitzer conductivity to determine $T_{e}$ and assume $n_{\text {sep }}=\bar{n} / 3$ we find that $\alpha$ so defined is given by

$$
\tilde{\alpha}=1.92 \cdot 10^{-26} R^{9 / 7} a^{-2 / 7} q_{c y l}^{18 / 7} \bar{n} \lambda_{q}^{-9 / 7} B_{t}^{-2} P_{S O L}^{2 / 7}\left(1+\kappa^{2}\right)^{-1 / 7}\left(\frac{Z_{e f f}+4}{5}\right)^{2 / 7}
$$

Figure 5 shows that this parameter, evaluated for the data shown in Figure 1, rises rapidly with $f_{G W}$, largely independently of other parameters, as can also be seen for the theory by substituting the HD formula for $\lambda_{q}$ into the above equation.

$\tilde{\alpha}_{H D}=0.123 f_{G W} \frac{n_{s e p}}{\bar{n}}\left(\frac{q_{c y l} R B}{a} P_{S O L}\right)^{1 / 8}\left(1+\kappa^{2}\right)^{3 / 2}\left[\frac{2 \bar{A}}{(1+\bar{Z})}\right]^{-9 / 16}\left(\frac{Z_{e f f}+4}{5}\right)^{1 / 8}$

For typical plasma parameters in this data set, this gives $\tilde{\alpha}_{H D} \sim(2-3) \cdot f_{G W}$.

These results are strikingly similar to measurements on C-Mod [22], which showed the measured $\alpha$ in the SOL to be independent of $B$ and $I_{p}$ at a given $f_{G W}$, but rising with $f_{G W}$ until detachment occurred. Experimental work on DIII-D [24] suggests an increase in $\tilde{\alpha}$ with density, which also may be halted as the plasma detaches from the target.

These observations suggest the idea that the high-density limit of the H-Mode, and of the Greenwald limit more generally, could be caused by the onset of MHD-driven turbulence in the SOL, rather than in the core or the pedestal. When the plasma falls out of the H-Mode due to this turbulence it could be close to the L-Mode density limit as well, depending on the specific scaling of the L-Mode SOL width with density. 
If we assume that the SOL $\beta$ limit is defined by criticality to MHD instability,

characterized as $\tilde{\alpha}_{c r i t} \sim C_{\alpha}\left(1+\kappa^{2}\right)^{\gamma}$ we find

$$
f_{G W, c r i t}=8.13 \cdot C_{\alpha} \frac{\bar{n}}{n_{\text {sep }}}\left(\frac{q_{c y l} R B}{a} P_{S O L}\right)^{-1 / 8}\left(1+\kappa^{2}\right)^{\gamma-3 / 2}\left[\frac{2 \bar{A}}{(1+\bar{Z})}\right]^{9 / 16}\left(\frac{Z_{\text {eff }}+4}{5}\right)^{-1 / 8}
$$

which is of order unity, and shows little variation with key parameters.

More extensive theoretical and experimental work is required to determine if strong instability is induced at the separatrix when the values of $\tilde{\alpha}$ being approached in Figure 5 are exceeded. In order to investigate the Greenwald limit itself, rather than phenomena associated with the H-Mode power requirement, it will be necessary to assure that sufficient power is injected during a density scan that divertor detachment or poorer confinement near the $\mathrm{H} \rightarrow \mathrm{L}$ transition do not occur before the Greenwald density limit is reached.

Finally, it is interesting to note that the HD mechanism would not necessarily be expected to be present in stellarators, with their tangled field lines near the separatrix. The field topology, rather than the complex cross-field particle drifts, could easily be the dominant determinant of the SOL width in such devices. In the picture developed here, this would explain the absence of the Greenwald limit in stellarators. It would also suggest that the SOL width should scale with the system size in stellarators, a favorable feature that should be investigated experimentally.

\section{Conclusions}

The HD model has been surprisingly successful at matching experimental data in both HMode divertor and L-Mode limiter tokamak plasmas, suggesting that a hot thin layer of plasma with width of order twice the drift excursion distance during a poloidal sound transit time, $2(a / R) \rho_{p}$, is a ubiquitous feature of tokamaks. The theoretical implications of this should be explored further with simulation codes and detailed local measurements, in order to 
elucidate the underlying mechanisms, including a possible role in setting the density limit. The practical implications for ITER and a future Demo are significant for the design of plasma-facing components. This analysis should not be directly applicable to stellarators, where the divertor width may be set by the magnetic topology. This may explain the absence of a density limit in stellarators, and suggest a more favorable size scaling of the SOL width.

\section{Acknowledgments}

The author thanks Richard Pitts for his leadership in the effort to uncover the physics of the near SOL in limiter plasmas. He also thanks A. Chankin for helpful discussions. For early access to data, the author thanks Jan Horaçek, Renaud Dejarnac and the COMPASS team, Peter Stangeby and the DIII-D team, Brian LaBombard and the C-MOD team, Fedorici Nespoli and the TCV team, and Gilles Arnoux and the JET team. This work supported by DOE Contract No. DE-AC02-09CH11466.

\section{References}

[1] R.J. Goldston, Nucl. Fusion 52 (2012) 013009

[2] T. Eich et al., Phys. Rev. Lett. 107 (2011) 215001

[3] T. Eich et al., Nucl. Fusion 53 (2013) 093013

[4] R.J. Goldston, T. Eich, IAEA Fusion Energy Conference 2012, TH/P4-19

[5] A.V. Chankin, D.P. Coster, Journal of Nucl. Mat. 438 (2013) S463

[6] R.J. Goldston, Journal of Nucl. Mat. 438 (2013) S372

[7] A.V. Chankin et al., Plasma Phys. Control. Fusion 48 (2006) 839

[8] J.D. Callen et al., Nucl. Fusion 50 (2010) 064004

[9] W. Fundamenski et al., Nuclear Fusion 51 (2011) 083028

[10] D.G. Whyte et al., Journal of Nucl. Mat. 438 (2013) S435 
[11] P. Stangeby, “The Plasma Boundary of Magnetic Fusion Devices”, IOP Publishing Ltd., London, 2000, Chapter 5, Section 4

[12] M. Jaworski, A. Khodak and R. Kaita, Plasma Phys. Control. Fusion 55 (2013) 124040

[13] R. Cabrera-Trujillo, J.R. Sabin, E. Deumens, Y. Öhrn, Phys. Rev. A 78 (2008) 012707

[14] A.V. Chankin et al., Journal of Nucl. Mat. 145-147 (1987) 789

[15] T. Denner, K.H. Finken, G. Mank, N. Noda, Nucl. Fusion 39 (1999) 83

[16] G. Arnoux et al., Nuclear Fusion 53 (2013) 073016

[17] J. Horaçek et al., this conference; Inner wall IR camera data from recessed limiter.

[18] R. Dejarnac et al., this conference; Inner wall probe data.

[19] A. Scarabosio et al., J. Nucl. Mat., 438 (2013) S426

[20] F. Nespoli et al., this conference, Inner wall IR camera data. Data selected to reduce error associated with finite IR coverage: $\lambda_{f a r}<3 R_{\max }-45 \mathrm{~mm}$ based on simulated error analysis.

[21] P. Stangeby et al., this conference, Inner wall swing probe data.

[22] B. LaBombard, private communication, SOL probe data.

[23] B. LaBombard et al., Phys. of Plasmas, 18 (2011) 056104

[24] M. Makowski, this conference 
Figure Captions

Figure 1. Experimental measurements of the inter-ELM exponential feature, $\lambda_{q}$, in a range of low-gas-puff H-Mode tokamaks, plotted against the Heuristic Drift model. [4]

Figure 2. Regression coefficients and their $1-\sigma$ error bars for data shown in Figure 1, compared with HD model scalings. $R / a \sim 3$ includes ASDEX-Upgrage, C-Mod, DIII-D and JET.

Figure 3. 2-Point Model calculation of ITER OMP and target temperatures as function of the fraction of dissipated power.

Figure 4. Experimental measurements of "near" exponential feature in a range of tokamaks. [16 - 23] plotted against the Heuristic Drift model.

Figure 5. Estimated MHD $\alpha$ parameter vs. Greenwald parameter, for data in Figure 1. 
Figure 1

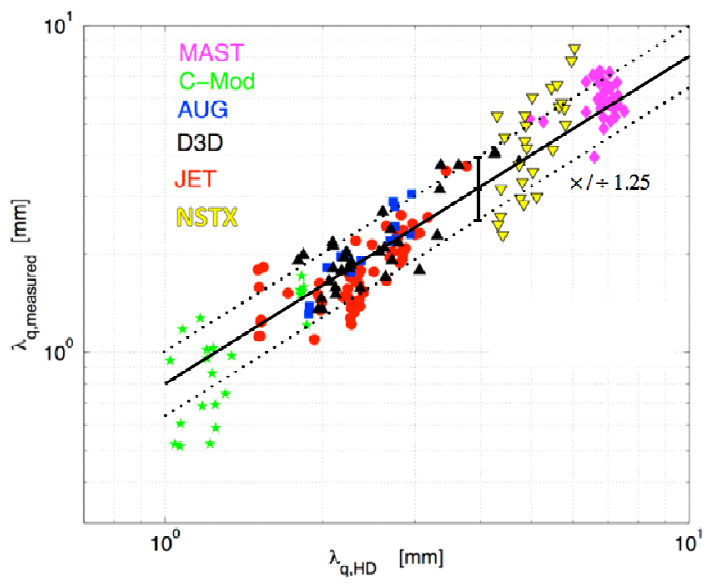


Figure 2

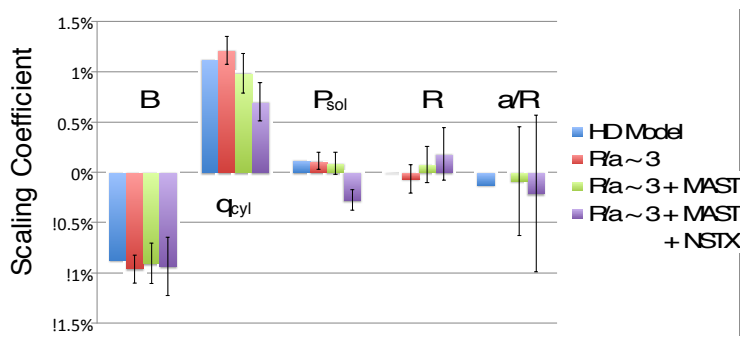


Figure 3

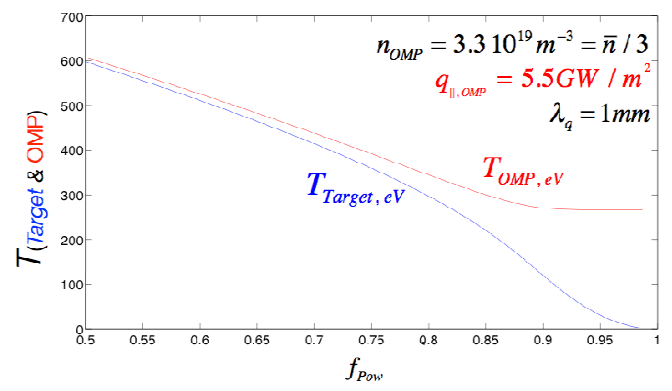


Figure 4

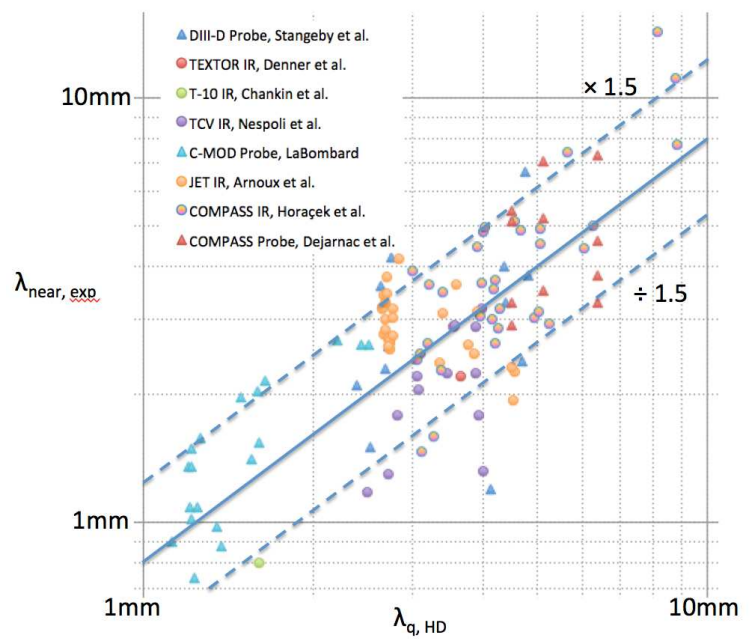


Figure 5

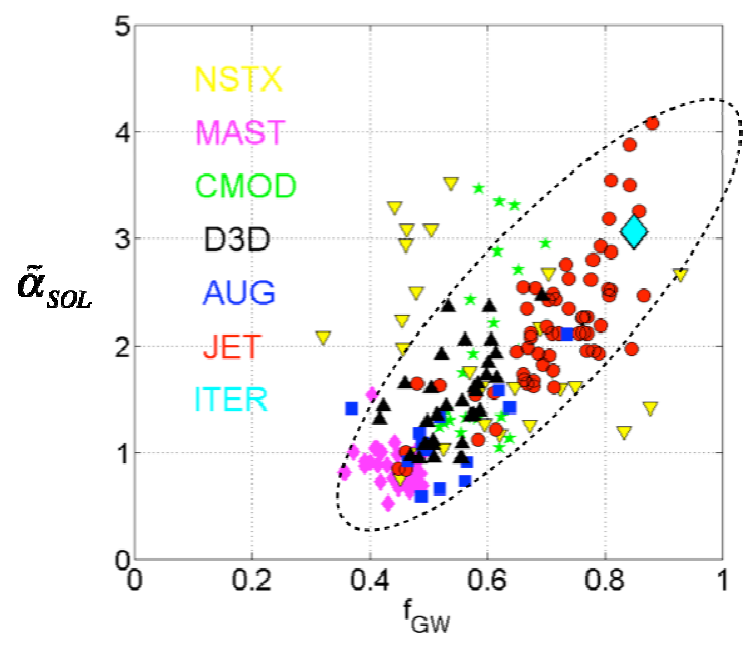

